\title{
ICU Communication Representation: Clinician-Clinician and Clinician- Computer Interactions
}

Saif Khairat ${ }^{1 *}$ and Yang Gong ${ }^{1,2}$

${ }^{1} \mathrm{MU}$ Informatics Institute, University of Missouri, Columbia, USA

${ }^{2}$ Health Management and Informatics, University of Missouri, Columbia, USA

\begin{abstract}
Objective: The aim of this study was to analyze clinical communication factors and interruptions and to develop clinician-clinician and clinician-computer knowledge representation models.

Methods: An ICU observational study was combined with medical error reported cases to address the above questions. Researchers shadowed the ICU team, for 55 hours during patient rounds, to capture 6 main communication factors. Simultaneously, a systematic literature search was conducted to identify and extract reported medical error cases caused by clinical communication problem. The search included patient safety data banks, literature databases,
\end{abstract} newspaper, and reported lawsuits.

Results: Out of 242 reported communication errors, 100 cases resulted in active errors while only 13 cases resulted in 13 near misses; most of those errors were reported in journal articles $(n=302)$. As to the observation data, the most frequent communicator during ICU patient rounds was the Attending Physicians. The ratio of interruptions caused by clinicians to technology-aided devices was 3:1 per patient visit. The mean frequency of an Attending Physician interacting with a computer was once per patient visit. Analyzing data from both sources, two communication models representing the clinical communication framework were developed.

Conclusion: Clinical communication is essential for effective health care delivery and for improved care outcomes. To further understand clinical communication, primary and secondary data were collected and analyzed and as a result, clinician-clinician and clinician-computer interaction models were developed to put into perspective the various factors involved in the communication process among care givers.

Keywords: Communication Models; Patient Safety; Intensive Care Unit; Medical Errors.

\section{Introduction}

The impact and cures of inadequate health communication is a core interest in the medical research field. Numerous attempts have examined ways to enhance communication between providers and patients[10,12,14,20,21]; even though this type of communication is of utmost importance, yet, there is another type of communication that is as significant, namely, communication among care givers. Our work focuses on the communication patterns among the Intensive Care Unit (ICU) clinical team.

In health care, communication is considered the backbone of many basic and crucial tasks for instance in education, information dissemination, knowledge exchange, and decision making. Clinical communication has a pivotal role in the information flow cycle; scientific evidence shows that communication errors can cause significant morbidity and mortality rates [6]. For those reasons, strong clinical communication is paramount for better health care outcomes. Clinical communication refers to the exchange of ideas, messages or knowledge between two or more entities through verbal, nonverbal, written, and visual forms where entities represent clinicians or technology aided-devices components. In this section, we introduce the significance of clinical communication, current state of ICUs, and the need for clinical communication model.

\section{The significance of clinical communication}

Medical errors are a national nightmare that everyone, from care givers to patients, is trying to escape from. In 2010, the Office of Inspector General (OIG) at the Department of Health and Human Services reported that each month one out of seven Medicare patients is injured or killed by medical errors [18] . The same report states that medical errors cost $\$ 4.4$ billion in 2009 taxpayer dollars. The findings of the OIG report affirm the previous reports from the Institute of Medicine (IOM) which state that as many as 98,000 people die each year as a result of preventable errors [11]. This shows that medical errors have higher mortality rates exceeding other causes of deaths such as auto accidents and breast cancer. These statistics show that medical errors represent a heavy burden on the economy, and most importantly, the loss of life due to medical errors is definitely an unwanted consequence.

Clinical miscommunication, the failure or incompleteness of message exchange, is the lead cause behind $75 \%$ of medical errors and $82 \%$ of sentinel events $[8,11]$.Similarly, the Harvard Medical Practice study shows that inefficient communication isa significant factor in theoccurrence of medical errors [3].Therefore, the improvement of communication in health care will yield significantly positive results on the quality of care being delivered and also financially, by achieving lower costs and increasing revenue and profit.

*Corresponding author: Saif Khairat, MS, PhDc, MU Informatics Institute University of Missouri, Columbia, MO, 65201, USA, Tel: +1 (573) 529-1786; E-mail: sekfz5@mail.missouri.edu

Received October 25, 2011; Accepted November 21, 2011; Published November 25, 2011

Citation: Khairat S, Gong Y (2011) ICU Communication Representation: ClinicianClinician and Clinician-Computer Interactions. J Health Med Informat S1. doi:10.4172/2157-7420.S1-001

Copyright: (C) 2011 Khairat S, et al. This is an open-access article distributed under the terms of the Creative Commons Attribution License, which permits unrestricted use, distribution, and reproduction in any medium, provided the original author and source are credited. 


\section{The nature of and communication at ICUs}

Patients with life threatening conditions are admitted to the ICU and intensive medical interventions are carried out by the clinical team in a fast-paced environment [2]. The multidisciplinary and complex nature of care required in the ICU makes it a setting ripe for the frequent occurrence of medical errors. Annually, more than two million patients are admitted to urban U.S. ICUs, of which approximately 200,000 die each year [15] .Moreover, mortality rates in admitted ICU patients average 10-20\% in most hospitals [24]. The overwhelming environment, overloaded clinicians, and critically ill patients provide a major challenge for effective communication to take place.

Communication failures are considered the common cause in ICU adverse events, and calls for improved patient safety through better communication have been made $[16,23]$. Among the benefits of better communication is the reduction of patient harm, shorter length of stay, higher caregiver satisfaction, and reduced cost [25].Henceforth, we dedicate this research to studying clinical communication factors in various ICUs such as Pulmonary, Burn, and Cardiac.

\section{Current communication models}

Over the course of many centuries, humans have realized the necessity of communication in order to accomplish most affairs, if not all. In the past, the nature of communication was relatively straight forward; it included direct communication between people. This can be shown in most communication models such as the models of Shannon, Berlo, and Shamm. Those well-constructed models represent the basic methods of communication and do not include new communication mediums. We studied the strengths and limitations of each of the general models then, we measured the relevance of those models against our first clinical communication model [13] . Results showed that there is a gap between the design of previously proposed non-clinical models and typical clinical communication scenarios. Therefore, it is time for ICUs to have their own communication model that represents the framework in which clinicians interact and collaborate.

\section{Methods}

In order to develop an ICU communication model that can accurately represent communication events, there is a need to build a knowledge base that encompasses most communication patterns and habits. For that reason, we believe that there is a necessity to collect communication events from two main sources: (1) literature, and (2) observation. Our data collection plan combined previously reported communication failure cases and data generated from our observational study.

\section{Reported cases}

The Veterans Health Administration and the Department of Defense are the only two organizations that mandate error reporting [22]. So, error cases were collected from the Agency for Healthcare Research and Quality (AHRQ), literature databases, such as Ovid, newspaper and magazine articles, and lawsuits. We utilized the wellstructured and organized feature of AHRQ called Patient Safety Net (PSNet). PSNet is a national web-based resource that provides the latest statistics and essential resources on patient safety. In order to retrieve all communication cases reported in PSNet, we searched for "medical error cases", which in return, provided 723 reported cases. Then, we refined our research to only include all cases labeled with "communication improvement", the returned number of cases where
668 of which 414 were labeled "communication between providers". Those cases were gathered from various sources and some of the cases were labeled by error types; Table 1 shows the distribution of cases.

Among the reported error incidents, a case reported at the Pennsylvania Patient Safety Advisory report, which discusses how to improve telephone and verbal orders. A nurse received a verbal order from the physician for Zosyn. The patient had documented that they are allergic to penicillin; however, both clinicians were unaware that Zosyn is derived from penicillin. The pharmacy staff was out of the office for the day and the medication was obtained from the night cabinet; two doses were administered to the patient. The next day, the pharmacy notified nursing that Zosyn is prohibited for this patient and the medication was discontinued [19]. This incident shows the importance of feedback, Decision Support Systems (DSS), education, and other communication factors in order to avoid such medication errors. It is labor intense to locate and analyze each error case because of the absence of a unified structure to all the cases. In other words, the ability to utilize Natural Language Processing (NLP) algorithms remains modest, until the cases are formatted in a computer-readable structure.

\section{Observational study}

In order to develop a comprehensive ICU communication model, the analysis of reported error cases is not sufficient. We believe that conducting an observational study at the ICU will further strengthen our findings that will be included in the model. Therefore, we conducted a research study in critical units at the307-bed University of Missouri Hospital; which in 2011, according to American Hospital Association, had 35,671 emergency room visits and performed 6,284 inpatient surgeries [1] . This study does not aim at capturing ICU medical errors; rather, we aim at capturing communication patterns and behaviors that can potentially be the cause of medical errors.

The study was conducted by two researchers/observers, who are under health informatics training, in order to increase the validity of data captured. The study design included shadowing 3 attending physicians and their clinical team during patient rounds for two weeks per attending physician. The focus of the study is to observe and understand the communication between the attending physician and their team. During the two-week period, the researchers shadowed the team 6 times, as a repeated measure, with the following distribution:

- $\quad$ First day of the first week

- Two days during the weekend

- Two week days

- Last day of the second week

This distribution was suggested by ICU domain experts as well as by our research biostatistician. From a clinical point of view, the first and last day of the two-week period will capture the communication patterns during a chaotic first day and then, the more organized

\begin{tabular}{|l|l|l|l|}
\hline Resource Type & Frequency & Error Types & Frequency \\
\hline Book/Report & 14 & Active errors & 100 \\
\hline Clinical Guideline & 2 & Latent errors & 33 \\
\hline Journal Article & 302 & Near miss & 13 \\
\hline Newspaper/Magazine & 71 & Epidemiology of errors and & 96 \\
\hline Web Source & 4 & Adverse events & \\
\hline
\end{tabular}

Table 1: Break down of error-reported cases at AHRQ by resource and error types. 
communication found at the end of the rotation period. In addition, weekends provide a different pace and intensity than weekdays, which consequently might reveal interesting relationships. While shadowing the clinical team, each researcher utilized our ICU communication checklist that is designed to capture certain human-human and human-computer interactions.

\section{Results}

The ICU clinical team was shadowed for 6 weeks, approximately 55 hours, and during that period the study focused on 6 main communication categories. Those categories capture most important communication events that occurred during patient rounds; table 2 shows the breakdown of communication activities by the communicators, types of interruptions, and computer interactions. Communication from the Attending physician had the highest rate of frequency with 13 instances per patient visits. These types of communication from the Attending physician included teaching statements, requesting new tasks, inquiring about patient status, and providing information that new team members were not aware of. The second most frequent activity during rounds was feedback given from the Attending physician. The Attending physicians constantly provided verbal and non-verbal feedback to the person communicating, which showed statistically significant impact on the team's awareness and satisfaction levels, with a mean of 4.3 out of 5 . Next, the clinical team showed a significant information exchange rate with the Attending. We found an association between the frequency of information given by the clinical team and how the team rated their participation and satisfaction levels.

Furthermore, we captured the frequency of interruptions that occurred during rounds. We categorize the types of interruption into two categories: (1) clinician-related interruptions, and (2) technologyaided devices interruptions. We found that both types of interruptions occurred in almost all patient visits however, interruptions caused by clinicians occurred approximately 3 times during a patient visit while interruptions from technological devices occurred only once.

\section{Clinician-clinician communication}

While interacting, clinicians constantly exchange information and knowledge; this rather complicated process involves two or more communicators, communication factors, and communication events. A communicator is a clinician who interacts with other clinicians by

\begin{tabular}{|l|l|l|l|}
\hline Communication Activity & $\begin{array}{l}\text { Total } \\
\text { Frequency }\end{array}$ & $\begin{array}{l}\text { Average frequency } \\
\text { per study session } \\
(\mathrm{n}=18)\end{array}$ & $\begin{array}{l}\text { Average frequency per } \\
\text { patient visit (n=279) }\end{array}$ \\
\hline $\begin{array}{l}\text { Patient information } \\
\text { conveyed to Attending } \\
\text { Physician }\end{array}$ & 1571 & 87.28 & 5.63 \\
\hline $\begin{array}{l}\text { Feedback given by } \\
\text { Attending Physician }\end{array}$ & 2041 & 113.39 & 7.31 \\
\hline $\begin{array}{l}\text { Communication events } \\
\text { done by Attending } \\
\text { Physician }\end{array}$ & 3658 & 203.22 & 13.11 \\
\hline $\begin{array}{l}\text { Interruptions caused by } \\
\text { clinicians }\end{array}$ & 844 & 46.70 & 3.03 \\
\hline $\begin{array}{l}\text { Interruptions caused } \\
\text { by technology-aided } \\
\text { devices }\end{array}$ & 342 & 19.00 & 1.23 \\
\hline $\begin{array}{l}\text { Attending-Computer } \\
\text { interaction }\end{array}$ & 318 & 17.67 & 1.14 \\
\hline
\end{tabular}

Table 2: Frequency of clinical communication events. sending or receiving clinical information. Communication factors refer to tacit and explicit knowledge that affect the way a communicator formulates or interprets a message. Communication activities represent clinician's behaviors and technological instances that have an impact on communication. Figure 1 represents the clinician-clinician communication model where a clinician communicates with one or more fellow clinicians. The model shows communication factors and activities that were reported in literature and were observed during the observational study.

The communication factors form a knowledge base that shapes the way a clinician interacts with peers. Continuing our work towards further understanding factors that impact communication [13], there are six communication factors that are responsible for how a clinician creates and perceives a message. The level of education and training, and the years of experience a clinician acquired are considered essential factors in the way clinicians exchange information. The previously mentioned factors, when at similar levels, facilitate inter and intracommunication among clinicians since with more education and experience clinicians self-learn how to effectively communicate with their peers.

Considering a clinician's culture is key to reaching high levels of communication effectiveness. Initially, we referred to culture as the background and tradition of a clinician. For example, in some international cultures to agree to a statement the person shakes their head sideways; while in the U.S. A person nods their head to indicate acceptance or agreement. Based on experience, we added a new dimension to the definition of culture, which is the variance in clinical backgrounds and practices among clinical specialties. Finally, we have observed that the overwhelming flow of information and knowledge combined with long working shifts can cause an incorrect recall of events or information. For that reason, cognitive psychology plays an important role during clinician-clinician interaction.

Moving on to communication activities that occur during patient visits at the ICU, we have observed the occurrence of 6 main activities. Even though it is not frequent, multitasking by a receiver usually leads to the sender repeating the message, mostly when the sender requests feedback from the receiver. The other activities are considered as interruptions, which we define as the interference to an ongoing conversation resulting in a pause or an end to the conversation. Clinician interruptions occur when a question interrupts the conversation, or if team members who are not participating in the conversation engage in a side conversation. External interruptions refer to questions or statements from non-clinicians, such as a patient's family. During patient rounds, pagers and system alerts constituted most interruptions in the technology-aided devices category; nevertheless, their benefits cannot be understated towards patient safety and clinical workflow.

\section{Clinician-computer interaction}

Computer interactions are an undivided part of the overall communication process. The utilization of computer systems is clearly present in each patient visitation, figure 2 represents the ICU Clinician-Computer Interaction; the model discussed clinical communication from two perspectives, the clinician and computer. During our observation, we summarized the usability of clinicians to computer systems into storing and retrieving patient information, request new tasks, viewing various types of imaging. In order for a clinician to effectively interact with a computer system, the clinician must receive adequate system training. We define adequate training as 


\section{Clinician-Clinician Interaction}

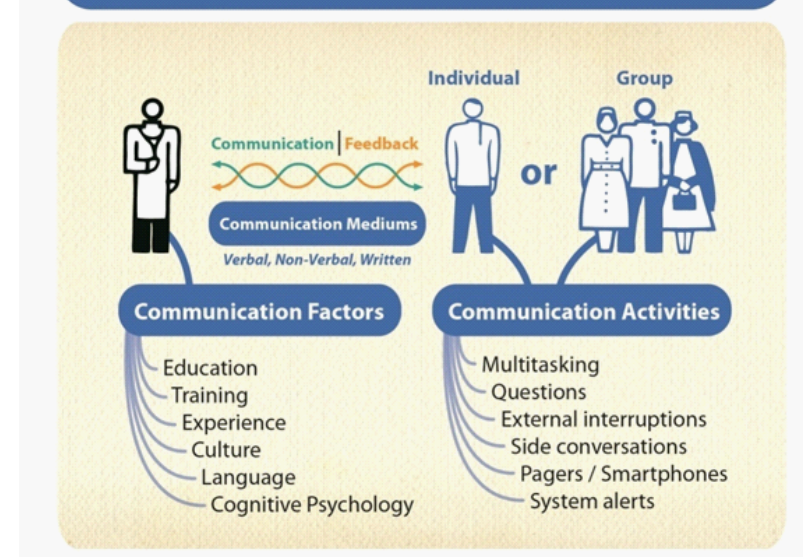

Figure 1: Clinical communication model with an emphasis on Clinician-Clinician interaction.

\section{Clinician-Computer Interaction}
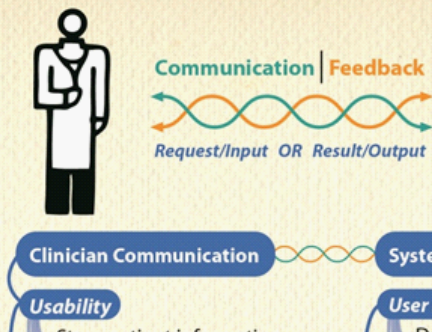

Store patient information Review patient information - Request tasks/order sets - View imaging

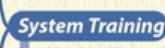

Navigation

Information storage \& retrieva

- Information interpretation

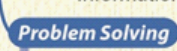

Workaround alerts

Editing

- Alternative solutions to

fatal errors
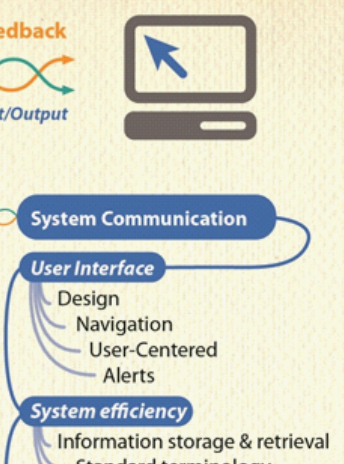

Information storage \& retrie

Standard terminology

Feedback

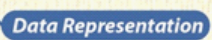

Visual representations

Quality

- Accuracy

Real-Time

Figure 2: Clinical communication model with an emphasis on Clinician-Computer interaction.

the level of which a clinician can fully and correctly use the available system functionalities. Clinicians should be able to navigate the system efficiently by finding the intended information in the shortest and quickest route. Moreover, different clinical systems provide different data interpretations, and clinicians should be able to correctly interpret those formats in a way that is least confusing. Through an effective training, the cognitive gap between system capability and users' expectation should be minimized. Furthermore, in the case of unusual system behavior, it is necessary that clinicians provide problem solving skills. For instance, in the case of error alerts to users, clinicians avoid delays by working around minor or temporary errors either through the system or in person. Also, in the rare instance of fatal errors, such as computer crashing, clinicians should have problem solving skills that would provide an alternate solution to the situation until the system is up again.

On the other hand, implementing a computer system in the ICU should focus on three main areas: (1) graphical user interface, (2) efficiency, (3) data representation. A user friendly interface can be reached through simple design and consistent navigation. Clinician's opinion on the interface must be fully considered when developing the system, their opinion on the design and navigation is important; however, their similarly important opinion on the functionality and effectiveness must not be left out. Furthermore, an efficient data storing and retrieving process that is error-free and easy to use is evident in critical units. Due to the labor intensive environment, clinicians are more susceptible to typos while requesting a patient's medical record; hence, system feedback is an essential error-checking procedure prior to executing a command. Also, standard terminology throughout the system is necessary to avoid inconsistencies and confusion. Finally, meaningful and reliable data representation is necessary for effective clinician-computer interaction. Visual representations are easier to read and interpret than many numeric values. Also, it is necessary for the system to represent real-time, accurate patient data, and high imaging quality.

\section{Discussion}

The primary purpose of this study research is to use qualitative approach to investigate communication events, interruptions, and interactions that the clinical team experienced within the context of critical care. The data collected through reported cases and observation resulted in 3 proximate outcomes that assist further understanding of the problem: 1) frequency of occurrence of instances, 2) representation of clinician-clinician interaction, 3) representation of cliniciancomputer interaction.

\section{Communication instances frequency}

We identified 6 main communication factors that repeatedly occurred during team communication with the Attending Physician: 1) patient information conveyed to the Attending, 2) feedback provided to the Attending, 3) frequency of communication done by the Attending, 4) interruptions by the team, 5) interruptions by technological devices, 6) Attending's interaction with computers. Table 2 shows that the majority of communication done in the ICU is done for the purpose of either giving or receiving information, which agrees with the findings of Coiera and Moss [7,17] with regards to the operating room and emergency departments. Communication done by the Attending to the team members was more than double the communication done by the team members to the Attending, which shows that more information is being transferred from the Attending which is logical due to the role and responsibilities of the Attending towards the patients and the team. Moreover, the Attending provided feedback, verbal and nonverbal, 1.5 more times than the communication instances they received from the team. This shows the persistency of the Attending to acknowledge and confirm the messages they received and also, an indication to the team to follow the same habit of clearly showing their understanding of a given statement.

A significant number of interruptions were observed during communication. The frequency of an interruption caused by clinician 
was approximately triple the frequency of an interruption caused by technology-aided devices.This ratio agrees with the findings of Patel at al., which suggests that human interruptions are usually twice as frequent to technological interruptions [4] .From observation, we identify that human interruptions, even though more frequent, can be controlled by increase in awareness and training, the reason for this belief is that all interruption variables, such as the interrupter, are present in the room. However, interruptions caused by technologies, such as telephones and pagers, might be harder to control since the interrupter is not on-site; nevertheless, options such as putting personal cellphones on silent could minimize this frequency.

\section{Clinicians interaction}

When clinicians communicate numerous factors are taken into consideration which makes the communication process complicated. We identified that communication among clinicians is affected tacit knowledge and external activity. The way a clinician was trained, the level of education, and the years of experience shape how they formulate or perceive a message. During the study we observed that clinicians with more of the previously mentioned factors can more accurately articulate their messages. Similarly, there is a language factor which represents two aspects: The first is good use of English for non-native speakers, and secondly, the use of standardized clinical terminology during communication. This agrees with the findings of a study surveying 64 members of the National Association of School Nurses, which suggested that the use of standardized terminology among nurses reduces symptoms after intervention, and enhances patient safety [5].

External factors seem to limit the communication process rather than facilitate. The frequent occurrence of side conversations, pager and computer alerts, and multitasking presents a disruption to the ongoing conversation and the result was a request to repeat, or a question aimed at continuing the conversation. We also observed team members multitasking during communication, while the justification is understood, the consequence of multitasking can range from mishearing to executing the wrong order and hence, there is a higher chance for medical errors.

\section{Clinicians and computers}

While shadowing the clinical team, several rare instances of human-computer interactions occurred. When reviewing the latest $\mathrm{X}$-Ray for an ICU patient, the image was hard to read and interpret and the Attending reported that the quality of imaging was of fair quality and better representation and quality is needed. Another instance, during patient rounds the Attending requested the medical record of the patient to be retrieved from the system, upon retrieval, the resident notified the Attending of the patient information; however, the Attending realized that the information is incorrect. The resident incorrectly typed the wrong information and the system retrieved the incorrect medical record.

When representing the communication process between clinicians and computers, there are two dimensions to highlight: 1) the user, 2) the computer system. Users must have comprehensive understanding of the system, including usability, and correctly storing and retrieving information, and problem solving skills. As for computer systems, the most important feature is to design a clinician-centered system that will provide convenient design and functionality that suits the needs of clinicians.

\section{Future direction and limitations}

Studying ICU communication is a tedious and labor intense activity, and affording adequate human resources to study this important phenomenon is a major limitation to this study. In this study we focused on the Attending physician, since they are at the top of the hierarchy; however, in future work we aim to study other clinical roles such as Fellows, Residents, and Registered Nurses etc. Another limitation of the study is that it was carried out during morning rounds, which are communication intense and interaction diverse;however, there is a need to broaden this research to include other sessions such as handoff sessions and morning meetings, which will be included in future studies.

\section{Conclusion}

Without a doubt, the significance of communication in health care is pivotal with regards to better care and enhanced patient safety. In this article, we further studied clinical communication by analyzingdata from literature and by observation. The focus of the study was to further understand key communication factors and activities that occur during conversation. Based on reported error cases and observation data, we proposed two ICU clinical communication models with a focus on Clinician-Clinician and Clinician-Computer interactions. This initial attempt, to our best knowledge, to represent ICU clinical communication is a significant step towards an ultimate goal of this research of building an exhaustive clinical communication ontology, which is consistent with our early work [9]. Along with the communication models, the ontology will serve as an educational tool for clinicians, and we aim to utilize it in medical error reporting system in order to increase the quality of reported error cases. Our efforts aim at reaching better understanding of the clinical communication framework in order to decrease medical errors, enhance healthcare quality, and thus, improving patient safety.

\section{References}

1. AHA: American Hospital Association Hospital Statistics 2011 Edition, Retrieved August 28, 2011.

2. Andrews LB, Stocking C, Krizek T, Gottlieb L, Krizek C, et al. (1997) An alternative strategy for studying adverse events in medical care. Lancet 349 : 309-313.

3. Brennan T A, Leape LL, Laird NM, Hebert L, Localio AR, Lawthers AG, et al ( 2004) Incidence of adverse events and negligence in hospitalized patients: results of the Harvard Medical Practice Study I. Quality and Safety in Health Care 13: 145-151.

4. Brixey J J, Tang Z, Robinson DJ, Johnson CW, Johnson TR, et al.(2008): Interruptions in a level one trauma center: A case study. International Journal of Medical Informatics 77: 235-241.

5. Cavendish R (2001) The Use of Standardized Language to Describe Abdominal Pain. J Sch Nurs 17: 266-273.

6. Coiera E (2000) When Conversation Is Better Than Computation. J Am Med Inform Assoc 7:277-286.

7. Coiera, Enrico W., Jayasuriya, Rohan A., Hardy, Jennifer, Bannan, Aiveen, \& Thorpe, Max E. C.: Communication loads on clinical staff in the emergency department. 9.from http://ovidsp.ovid.com/ovidweb.cgi?T=JS\&PAGE=referenc $\mathrm{e} \& \mathrm{D}=\mathrm{med} 4 \& N E W S=N \& A N=12056992$, Retrieved 0400714, m26, 176, 2002

8. Commission, The Joint: Sentinel Event Data Root Causes by Event Type2004Fourth Quarter 2010. Retrieved July 27, 2011.

9. Gong Y, Zhu M, Li J, Turley J \& Zhang J (2007) Clinical communication ontology for medical errors. AMIA Annu Symp Proc 129: 1007-1012. 
Citation: Khairat S, Gong Y (2011) ICU Communication Representation: Clinician-Clinician and Clinician-Computer Interactions. J Health Med Informat S1. doi:10.4172/2157-7420.S1-001

10. Haughney J, Sims E, Holohan J, Ryan D, Price D ( 2010) Improving clinicianpatient communication in asthma: the HARP project. Allergy 65: 413-414.

11. IOM: To Err Is Human: Building a Safer Health System: The National Academies Press, Washington, 1999.

12. Kenny DA, Veldhuijzen W, Weijden T, LeBlanc A, Lockyer J, Légaré $F$, et al. (2010) Interpersonal perception in the context of doctor-patient relationships: A dyadic analysis of doctor-patient communication. Soc Sci Med 70: 763-768.

13. Khairat S, Gong Y (2010) Understanding effective clinical communication in medical errors. Stud Health Technol Inform 160: 704-708.

14. Knopp R, Rosenzweig S, Bernstein E, Totten V (1996) Physician-Patient Communication in the Emergency Department, Part 1. Acad Emerg Med 3: 1065-1076.

15. LeapFrog: The Leapfrog Group's Patient Safety Practices 2003: The Potential Benefits of Universal Adoption. Retrieved July 27, 2011, 2003.

16. Leonard M, Graham S, Bonacum D (2004) The human factor: the critical importance of effective teamwork and communication in providing safe care. Qual Saf Health Care 13: i85-i90.

17. Moss J, Xiao Y (2002) A comparison of communication needs of charge nurses in two operating room suites. Proc AMIA Symp 543-547.
18. OIG: Adverse Events in Hospitals: National Incidence Among Medicare Beneficiaries. Retrieved July 27, 2011, 2010.

19. Improving the safety of telephone or verbal orders (2006) PA PSRS Patient Saf Advis 3: 3-7.

20. Street RL Jr, Makoul G, Arora NK, Epstein RM (2009) How does communication heal? Pathways linking clinician-patient communication to health outcomes. Patient Educ Couns: 74: 295-301.

21. Street RL Jr, Slee C, Kalauokalani DK, Dean DE, Tancredi DJ, Kravitz RL (2010) Improving physician-patient communication about cancer pain with a tailored education-coaching intervention. Patient Educ Couns 80: 42-47.

22. Weeks WB, Bagian JP (2000) Developing a culture of safety in the Veterans Health Administration. Eff Clin Pract 3: 270-276.

23. Wright D, Mackenzie SJ, Buchan I, Cairns CS, Price LE (1991) Critical incidents in the intensive therapy unit. Lancet 338: 676-678.

24. Zimmerman JE, Wagner DP, Draper EA, Wright L, Alzola C, et al. ( 1998) Evaluation of Acute Physiology and Chronic Health Evaluation III predictions of hospital mortality in an independent database. Crit Care Med 26: 1317-1326.

25. Zwarenstein M, Reeves S (2002) Working together but apart: barriers and routes to nurse--physician collaboration. Jt Comm J Qual Improv 28: 242-247. 